\title{
Novel dehydropeptide-based magnetogels containing manganese ferrite nanoparticles as antitumor drug nanocarriers
}

\author{
Sérgio R. S. Veloso, ${ }^{a}$ Carlos A. B. Magalhães, ${ }^{a}$ Ana Rita O. Rodrigues, ${ }^{a}$ H. Vilaça, ${ }^{b}$ Maria-João R. P. \\ Queiroz, $^{b}$ J. A. Martins, ${ }^{b}$ Paulo J. G. Coutinho, ${ }^{a}$ Paula M. T. Ferreira, ${ }^{b}$ and Elisabete M. S. \\ Castanheira $^{\mathrm{a},{ }^{+}}$
}

\begin{abstract}
Novel dehydropeptide-based magnetogels, based on the hydrogelators Npx-L-Phe-Z- $\triangle \mathrm{Abu}-\mathrm{OH}, \mathrm{Npx}-\mathrm{L}-\mathrm{Trp}-\mathrm{Z}-\mathrm{\Delta Phe-OH}$ and Npx-L-Ala-Z- $\Delta$ Phe-Gly-L-Arg-Gly-L-Asp-Gly-OH and containing manganese ferrite nanoparticles (diameter around $20 \mathrm{~nm}$ ), were prepared and characterized. TEM and FTIR measurements showed that the magnetogels maintain the fibrous structure of the neat hydrogels, with fibres of $c a .20 \mathrm{~nm}$ average width (generally in the range $10-30 \mathrm{~nm}$ ) and a few conformational changes relative to the neat hydrogels.

The magnetogels were tested as nanocarriers for two potential fluorescent antitumor drugs, a thienopyridine derivative and the natural compound curcumin. FRET (Förster Resonance Energy Transfer) from the gels aromatic moieties (energy donors) to the fluorescent drugs (energy acceptors) and fluorescence anisotropy measurements confirmed the incorporation of both drugs in the magnetogels matrices. The transport of both drugs loaded in the magnetogels into membrane models (small unilamellar vesicles) was assessed by FRET between the fluorescent drugs and the dye Nile Red. The magnetogel possessing the RGD sequence was the most promising for delivery of the thienopyridine derivative, while the three magnetogels showed to be suitable for the delivery of curcumin.
\end{abstract}

\section{Introduction}

Nanotechnology has been of uttermost interest for therapeutics in recent years, mainly focusing on the development of new drug delivery nanosystems, due to the need of reducing cytotoxicity, adverse side effects and improving delivery efficiency. One of such nanosystems are magnetic nanoparticles (MNPs), that can be made of any transition metal ( $\mathrm{Fe}, \mathrm{Ni}, \mathrm{Co}, \mathrm{Mn}$ ) and its oxides. ${ }^{1}$ Superparamagnetism, which generally occurs in MNPs sizes smaller than $30 \mathrm{~nm}$, is one of their noble properties, as it allows the direct control to a specific location by using a magnetic field gradient. ${ }^{1-4}$

Transition metal ferrites are a class of soft-magnetic nanoparticles with superparamagnetic behaviour and other suitable properties for biomedical applications, such as chemical stability and mechanical hardness. ${ }^{2-6}$ Within this class of magnetic nanoparticles, manganese ferrite nanoparticles are of particular interest for biomedical applications, as their magnetic susceptibility is higher than those of other transition metal ferrites. ${ }^{2,47-8}$ Apart from being easily synthesized by coprecipitation (without the need of calcination steps), ${ }^{2}$ they also show good biocompatibility ${ }^{3,8,9}$ and slight toxicity against HeLa cells, ${ }^{10}$ possessing also suitable properties as contrast agents in magnetic resonance imaging, drug delivery and hyperthermia. ${ }^{2,11}$

However, when it comes to in vivo application, tissue invasion and toxicity have to be taken into account. ${ }^{4,9}$ The latter can be surpassed by using an organic or inorganic shell and the former by using nanoparticles with diameters smaller or comparable to 50 $\mathrm{nm}$, so that it may possible for NPs to penetrate endothelial fenestrations. ${ }^{4,9,12}$ The need of a moderate retention time can be addressed if magnetic nanoparticles are combined with other systems like hydrogels, elastic tridimensional networks of watersoluble polymers, ${ }^{13,14}$ which cross-linking or entanglement retains a huge portion of solvent. ${ }^{15}$ The variety of usable polymers, combined with the versatility of producible physical forms ${ }^{13}$ and stimulus to activate their formation, ${ }^{11,14,16}$ allow attaining a large diversity of physical and chemical properties, adjustable to the needs. ${ }^{17}$ In addition, it is possible to control drug diffusion through the change of structure porosity or the hydrogelator affinity to aqueous environment and density of reticulate agents. ${ }^{13}$

Peptide-based hydrogels are highly biocompatible due to the large portion of water and physical-chemical similarity to the extracellular matrix, both in composition and mechanical properties, thus having a wide range of applications, such as tissue engineering, in vivo imaging and drug delivery. ${ }^{18-19}$ The gelation of this type of hydrogels is usually driven by the cooperative effect of weak intermolecular interactions: hydrogen bonding, hydrophobic and aromatic $\pi-\pi$ interactions. ${ }^{15,18-19}$ Nevertheless, the $\mathrm{pH}$ value, kinetics of $\mathrm{pH}$ drop, temperature, ionic strength and structure amphiphilicity also affect gelation. ${ }^{19-21}$

Peptide-based hydrogelators are usually protected in the $\mathrm{N}$-terminal with naphthaloyl and fluorenylmethoxycarbonyl (Fmoc) groups, but naproxyl can grant more advantages. ${ }^{19,22}$ The presence of naproxen or any other aromatic moiety in the molecular structure, not only may grant selectivity for a certain target, but also contributes for aromatic interactions and other non-covalent forces. ${ }^{23}$ Apart from that, the use of dehydroamino acids not only endow peptides with proteolytic stability, but also enhances naproxen selectivity to Cyclooxygenase 2 (COX-2) and add conformational restraints to the peptide backbone. ${ }^{19,22-23}$

Recently, a new peptide-based hydrogelator with RGD motif was reported and its potential as drug delivery system was assessed. ${ }^{24}$ The RGD sequence (Arg-Gly-Asp) is a peptide sequence displayed by extracellular matrix protein vitronectin, which epitopes are recognized by cell receptors that trigger responses like cell migration and differentiation. ${ }^{24}$ Self-assembly of such hydrogelators 
originates fibrils with the self-assembly module at the core and the pendant epitopes available for interaction with integrins, ${ }^{24}$ overexpressed in cancer cells. ${ }^{25}$ Therefore, the incorporation of nanoparticles into hydrogels, obtaining nanomagnetogels, will allow their concentration in the therapeutic sites by magnetic forces, allowing the guided transport of anticancer drugs, most of them toxic and with systemic side effects. Furthermore, the synergy between magnetically-guided drug delivery and hyperthermia makes these nanosystems highly promising. ${ }^{26,27}$

In this work, magnetic nanoparticles of manganese ferrite were prepared and incorporated into self-assembled biocompatible dehydropeptide-based hydrogels containing a Naproxen moiety. The corresponding hydrogelators were previously synthesized (structures in Table 1) and some of them preliminary evaluated as drug nanocarriers. ${ }^{19,23,24}$ A recently synthesized antitumor thienopyridine derivative ${ }^{28}$ (Fig. 1) and a model drug with anticancer potential (curcumin, also in Fig. 1) were used to test the new magnetogels as drug nanocarriers for cancer therapy. The thienopyridine derivative has shown a potent inhibition activity against human cancer cell lines, including MCF-7 (breast adenocarcinoma), $\mathrm{NCl}-\mathrm{H} 460$ (melanoma) and A375-C5 (non-small cell lung cancer). ${ }^{28}$ Besides, this compound presents a very low affinity for the multidrug resistance protein MDR1, that promotes drug resistance in cells. ${ }^{29}$ Curcumin is a natural polyphenolic compound obtained from Curcuma longa roots and known for its role in immune system pathways and intracellular regulation. ${ }^{30-32}$ These properties, together with the lack of toxicity at high doses, makes curcumin as a promising therapeutic agent for cancer and neurodegenerative disorders, among others. ${ }^{31-33}$ However, curcumin is poorly water soluble and with low bioavailability, easy to metabolize and to systemic elimination, making it difficult for use in cancer treatment. ${ }^{33,34}$ Thus, considering the antitumor potential of both compounds, they were incorporated in the developed magnetogels, considering future applications of these nanosystems in oncological therapy.<smiles>CC(=O)c1sc2cc(Nc3nc4ccccc4s3)cnc2c1N</smiles>

Thienopyridine derivative<smiles>COc1cc(/C=C/C(=O)/C=C(O)/C=C/c2ccc(O)c(OC)c2)ccc1O</smiles>

Fig. 1 Structure of the antitumor thienopyridine derivative (methyl 3-amino-6-(benzo[d] thiazol-2-ylamino)thieno[3,2-b]pyridine-2carboxylate) and of the model drug curcumin.

\section{Experimental}

Preparation of manganese ferrite nanoparticles. Manganese ferrite nanoparticles were synthesized by the co-precipitation method in a total solution volume of $5 \mathrm{~mL}$, as described by Rodrigues et al. ${ }^{2}$ Initially, a mixture of $500 \mu \mathrm{L}$ of $\mathrm{MnSO}_{4} \cdot \mathrm{H}_{2} \mathrm{O} 0.5 \mathrm{M}$ and $500 \mu \mathrm{L}$ of $\mathrm{FeCl}_{3} \cdot 6 \mathrm{H}_{2} \mathrm{O} 1 \mathrm{M}$ was prepared and added, drop by drop, to an aqueous solution with $612 \mu \mathrm{L}$ of $\mathrm{NaOH} 50 \%$ at $90^{\circ} \mathrm{C}$ and constant magnetic stirring. After $2 \mathrm{~h}$ at $90{ }^{\circ} \mathrm{C}$, nanoparticles were formed and purification was carried out by repeated centrifugations being dispersed in deionized water, following by drying at $100^{\circ} \mathrm{C}$.

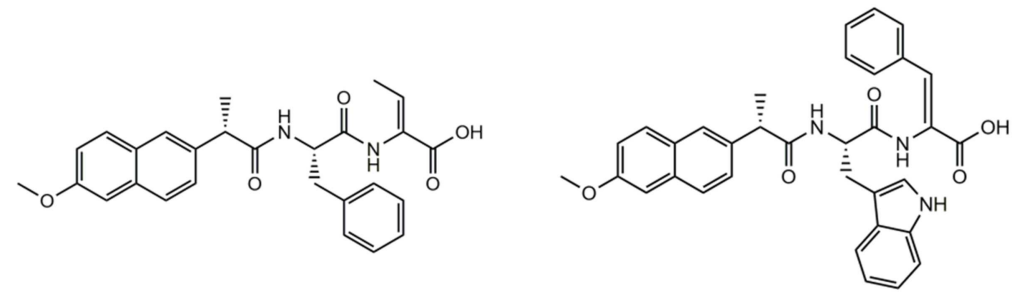

Npx-L-Phe-Z- $\triangle$ Abu-OH (H1)

Npx-L-Trp-Z- $\triangle$ Phe-OH (H2)

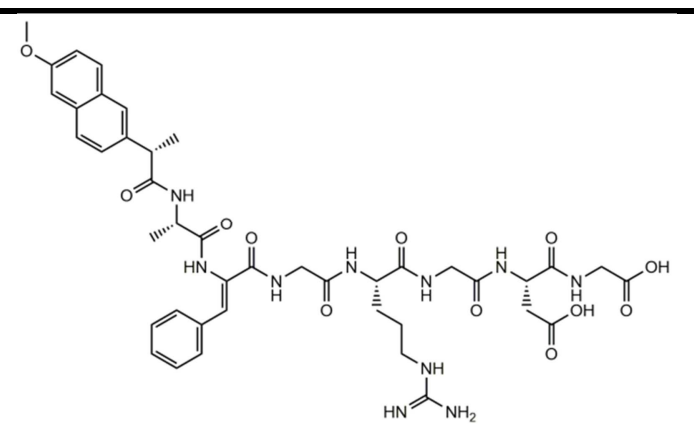

Npx-L-Ala-Z- $\Delta$ Phe-Gly-L-Arg-Gly-L-Asp-Gly-OH (H3)

\footnotetext{
${ }^{a}$ Npx: Naproxen; Phe: Phenylalanine; $\triangle$ Phe: Dehydrophenylalanine; Abu: Aminobutyric acid; $\triangle$ Abu: Dehydroaminobutyric acid; Ala: Alanine; Arg: Arginine; Asp: Aspartate; Gly: Glycine; Trp: Tryptophan.
}

Self-assembly of hydrogels and magnetogels. Hydrogels were prepared according to the previously determined gelification conditions $^{19,23,24}$ (Table 2) for a final volume of $300 \mu \mathrm{L}$. A stock solution of manganese ferrite nanoparticles $19 \mathrm{mM}$ was added to a final concentration of $0.1 \mathrm{mM}$. The weighted mass of hydrogelator was dissolved in the required $\mathrm{pH}$ buffer previously prepared from a sodium phosphate $0.1 \mathrm{M}$ solution and a mixed solution of citric acid $(0.05 \mathrm{M})$ and boric acid $(0.2 \mathrm{M})$, as described by Perrin and Dempsey. ${ }^{35}$ 
Table 2. Hydrogel preparation conditions. ${ }^{19,23,24}$

\begin{tabular}{lccc}
\hline \multicolumn{1}{c}{ Hydrogel } & pH & wt\% & GdL wt\% \\
\hline Npx-L-Phe-Z- $\triangle$ Abu-OH (H1) & 6 & 0.4 & -- \\
Npx-L-Trp-Z- $\Delta$ Phe-OH (H2) & 6 & 0.4 & 0.34 \\
Npx-L-Ala-Z- $\Delta$ Phe-Gly-L-Arg-Gly- & 6 & 0.4 & --- \\
-L-Asp-Gly-OH (H3) & & & \\
\hline
\end{tabular}

The gelation activation was carried out by a $\mathrm{pH}$ drop or by heating and cooling. The gelation of hydrogelators $\mathbf{H} \mathbf{1}$ and $\mathbf{H} \mathbf{3}$ was performed by solubilizing the hydrogelator in the required $\mathrm{pH}$ buffer (Table 2) at $60^{\circ} \mathrm{C}$, under stirring until the solution become translucid, and left cooling at room temperature. ${ }^{23,24}$ The hydrogelator $\mathbf{H} \mathbf{2}$ was dissolved in $\mathrm{pH}=10$ aqueous media by adding $\mathrm{NaOH} 1 \mathrm{M}$ solution and, then, glucono- $\delta$-lactone (GDL) was added to decrease $\mathrm{pH}$ homogenously to $\mathrm{pH}=6 .{ }^{19} \quad$ All hydrogels/magnetogels solutions were left standing at room temperature until gel phase was attained. The gelation occurred for all the hydrogels containing manganese ferrite nanoparticles.

Incorporation of drugs and transport into biomembrane models. The thienopyridine derivative or curcumin were incorporated through the addition of a small amount of drug ethanolic solutions to the hydrogelators solution before gelation (with already added magnetic nanoparticles), in a final concentration of $2 \mu \mathrm{M}$. To study the interaction between magnetogels and biomembrane models, small unilamellar vesicles (SUVs, used as membrane models) were prepared from soybean lecithin and cholesterol in a 7:3 ratio, by the ethanolic injection technique. ${ }^{36}$ An ethanolic solution of soybean lecithin and cholesterol was prepared and added drop-by-drop to ultrapure water (Milli-Q grade) under vigorous vortexing at room temperature for a total concentration of $1 \mathrm{mM}$. The lipid probe Nile $\operatorname{Red}^{37-39}(2 \mu \mathrm{M})$ was also incorporated in SUVs by mixing with the lipid solution before ethanolic injection.

All studies of drug incorporation and transport into SUVs were carried out by fluorescence-based techniques (fluorescence emission, FRET and fluorescence anisotropy).

\section{Spectroscopic measurements}

General methods. Fluorescence measurements were carried out using a Fluorolog 3 spectrofluorimeter, equipped with double monochromators in both excitation and emission, Glan-Thompson polarizers and a temperature controlled cuvette holder. Fluorescence emission spectra were corrected for the instrumental response of the system. Absorption spectra were recorded in a Shimadzu UV-3101PC UV-Vis-NIR spectrophotometer.

The fluorescence quantum yield, $\Phi_{s}$, can be determined by eqn (1) (standard method), ${ }^{40,41}$

$\Phi_{S}=\frac{\left(A_{r} F_{s} n_{S}^{2}\right)}{\left(A_{S} F_{r} n_{r}^{2}\right)} \Phi_{r}$

where $A$ is the absorbance at the excitation wavelength, $F$ the integrated emission area and $n$ is the refraction index of the solvents. Subscripts $r$ and $s$ refer to reference and sample compound, respectively. The absorbance value at excitation wavelength was always less than 0.1 , in order to avoid inner filter effects. L-Tryptophan in aqueous buffer solution $(\mathrm{pH}=7.2)$ was used as reference $\left(\Phi_{R}=0.14\right.$ at $\left.25^{\circ} \mathrm{C}\right) .{ }^{42}$

Fluorescence anisotropy measurements. The steady-state fluorescence anisotropy values, $r$, can be determined by eqn (2), ${ }^{43}$

$r=\frac{I_{V V}-G I_{V H}}{I_{V V}+2 G I_{V H}}$

where $I_{\mathrm{VV}}$ and $I_{\mathrm{VH}}$ are the intensities of the emission spectra obtained with vertical and horizontal polarization, respectively (for vertically polarized excitation light), $I_{\mathrm{HV}}$ and $I_{\mathrm{HH}}$ are the emission intensities obtained with vertical and horizontal polarization (for horizontally polarized excitation light) and $G=I_{\mathrm{HV}} / I_{\mathrm{HH}}$ the instrumental correction factor.

FRET measurements. The drug incorporation into magnetogels network and interaction with biomembrane models was investigated by Förster Resonance Energy Transfer (FRET).

FRET efficiency, $\Phi_{\text {FRET }}$, defined as the proportion of donor molecules that have transferred their excess energy to acceptor molecules, can be expressed by eqn (3), ${ }^{43}$

$\Phi_{\mathrm{FRET}}=1-\frac{I_{D A}}{I_{D}}$

where $I_{D A}$ and $I_{D}$ are the donor integrated fluorescence intensities in the presence and absence of acceptor, respectively. FRET efficiency can be related with intermolecular distance and Förster radius (critical diameter), $R_{0}$, through eqn (4), ${ }^{43}$

$\Phi_{\mathrm{FRET}}=\frac{1}{1+\left(\frac{R_{D A}}{R_{0}}\right)^{6}}$

Förster radius, $R_{0}$, the distance at which FRET efficiency is $50 \%$, can be determined by the spectral overlap, $J(\lambda)$, between the donor fluorescence emission and the acceptor absorption, according to eqn (5) and eqn (6) (with $R_{0}$ in $\AA$, $\lambda$ in nm, $\varepsilon_{A}(\lambda)$ in $\left.\mathrm{M}^{-1} \mathrm{~cm}^{-1}\right)^{43}$

$R_{0}=0.2108\left[\kappa^{2} \Phi_{D} n^{-4} J(\lambda)\right]^{1 / 6}$

$J(\lambda)=\int_{0}^{\infty} I_{D}(\lambda) \varepsilon_{A}(\lambda) \lambda^{4} d \lambda$

where $\kappa^{2}=2 / 3$ is the orientational factor assuming random orientation of the dyes, $\Phi_{D}$ is the fluorescence quantum yield of the donor in absence of energy transfer, $n$ is the refraction index of the medium, $I_{D}(\lambda)$ is the fluorescence spectrum of the donor normalized so that $\int_{0}^{\infty} I_{D}(\lambda) d \lambda=1$, and $\varepsilon_{A}(\lambda)$ is the molar absorption coefficient of the acceptor.

Transmission Electron Microscopy (TEM). HR-TEM images were recorded using a Transmission Electron Microscope JEOL JEM 2010F operating at $200 \mathrm{kV}$ coupled to an Electron Dispersive Spectroscopic analyzer (EDS) at C.A.C.T.I (Centro de Apoio Cientifico e Tecnolóxico á Investigación), Vigo, Spain. The samples were prepared according to the ammonium molybdate staining method. After preparation of the hydrogelators solutions (at $\mathrm{pH}=6 ; 0.12 \mathrm{wt} \%$ ), $20 \mu \mathrm{L}$ were mixed with $20 \mu \mathrm{L}$ of ammonium molybdate solution. The hydrogelator $\mathbf{H 2}$ required prior dissolution at $\mathrm{pH}=12$ followed by addition of $\mathrm{GdL}$. A drop of each sample was placed onto a TEM 400 mesh copper grid 
with Formvar/Carbon (ref. S162-4 from Agar Scientific), held by tweezers and left to dry.

The processing of TEM images was performed using ImageJ software, which consisted in enhancing local contrast and adjusting brightness followed by manual selection of particles and fibres.

X-Ray Diffraction. A conventional Philips PW 1710 diffractometer was used for X-ray diffraction (XRD) analyses, operating with $\mathrm{Cu} \mathrm{K}_{\alpha}$ radiation, in a Bragg-Brentano configuration.

FTIR measurements. Fourier Transform Infrared Spectroscopy (FTIR) measurements with Attenuated Total Reflection (ATR) were performed in a Bruker instrument.

Dynamic Light Scattering measurements. Mean hydrodynamic diameter, size distribution (polydispersity index) and zeta potential were measured using a Dynamic Light Scattering (DLS) NANO ZS Malvern Zetasizer equipment, at $25^{\circ} \mathrm{C}$, using a He-Ne laser of 633 $\mathrm{nm}$ and a detector angle of $173^{\circ}$. Five independent measurements were performed for each sample. Malvern Dispersion Technology Software (DTS) was used with multiple narrow mode (high resolution) data processing, and mean size $(\mathrm{nm})$ and error values were considered.

Molecular docking studies of the hydrogelators. The crystal structure of Mus musculus COX-2 expressed in Spodoptera frugiperda complexed with naproxen (Protein Data Bank (PDB) code: 3NT1) was used as the protein receptor model. The optimized geometries of the hydrogelators ground state were obtained from $a b$ initio molecular quantum chemistry calculations, with Gaussian 09 software $^{44}$ and use of a $6-31+G(d, p)$ basis set at the DFT B3LYP level of theory in gas phase. Docking of the receptor protein with the hydrogelators was performed using AutoDockTools-1.5.6 Software Genetic Algorithm. The calculation was set up to 20 runs, 270000 maximum number of generations, 2500000 maximum number of energy evaluations, and $60 \times 60 \times 60$ grid points for 3 NT1 with $0.375 \AA$ \& spacing. The macromolecule was kept rigid and ligand molecules were flexible. Due to the very high flexibility of the hydrogelator with the RGD motif (H3), first, a covalent map was used in the docking procedure, so that the oxygen atom of naproxen methoxy group was fixed at the same position where free naproxen was found to bind COX-2. The resulting conformation was subsequently redocked without the covalent map. The lowest docked energy conformation with naproxen moiety in the active site was chosen as the best conformation. Visualization of the complex protein-ligand interactions was analysed with PyMOL software.

\section{Results and discussion}

Molecular docking studies of the hydrogelators. Direct docking of the crystallographic naproxen was performed, and the best scoring pose (lowest binding energy) was superimposed over the original
PDB structure. The root mean square deviation was calculated to measure the docking prediction accuracy and an overall error of $0.61 \AA$ was obtained. Hydrogen bonding interactions and distances between naproxen carboxylate moiety and $\mathrm{Tyr}^{355}$ hydroxyl group and also $\operatorname{Arg}^{120}$ guanidinium moiety are presented in Figure $2 \mathrm{~A}$. The hydrophobic residues $\mathrm{Leu}^{531}, \mathrm{Val}^{349}, \mathrm{Ala}^{527}$ and $\mathrm{Val}^{523}$ are also shown in the active site, due to their role in the ligand stabilization through hydrophobic interactions. ${ }^{45,46}$

Cross docking between hydrogelators and COX-2 are shown in Figure 2B-D and followed the same procedure, except for H3 where an anchor at the naproxen methoxy group was found to be necessary due to the augmented flexibility of this hydrogelator. The obtained free energies of binding, intermolecular energy in the binding site and internal energy (both in bound and unbound state) that result from the AutoDock calculations are presented in Table 3. It can be observed that for all the studied hydrogelators the intermolecular energy is lower than that for naproxen. This indicates that the interactions of the additional (dehydro)amino acids with the protein are favourable. However, in the case of the RGD epitope, this additional stabilization is not too strong as to preclude its interaction with other intended targets. The observed less favourable binding energy of $\mathbf{H} \mathbf{3}$ is mainly due to the much higher loss of torsional free energy upon binding, when compared to the ones determined for $\mathbf{H 1}$ and $\mathbf{H 2}$ (Table 3). Nevertheless, the results point to an anti-inflammatory activity through interaction with COX-2 for these hydrogelators.

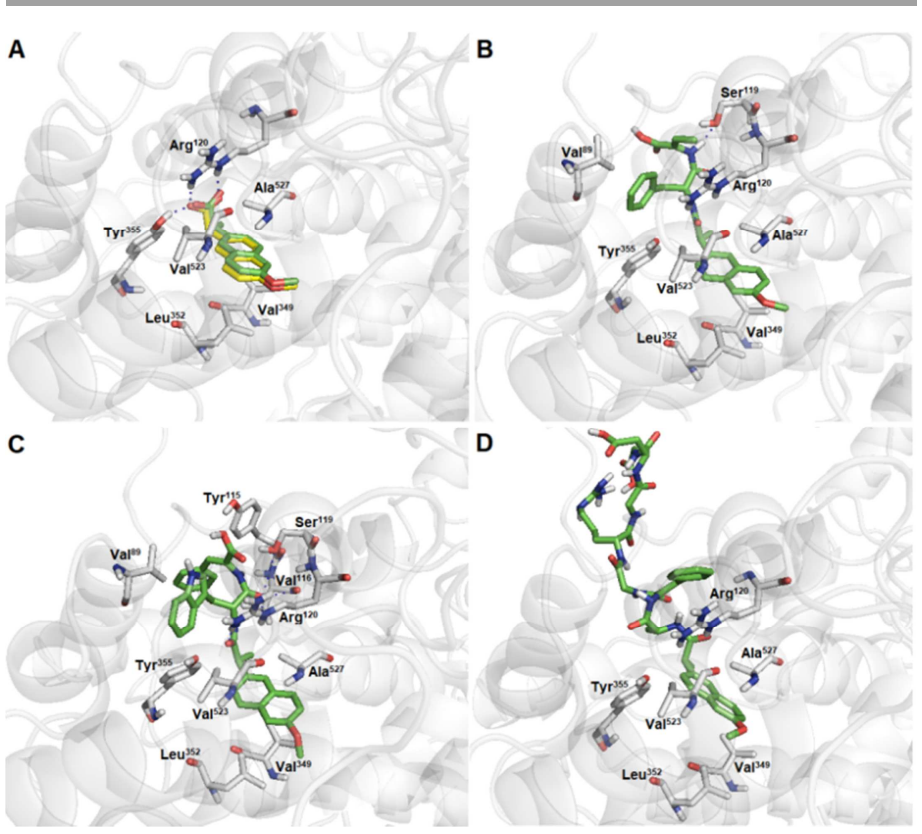

Fig. 2 Docking results for Naproxen (A) and for the hydrogelators H1 (B), $\mathbf{H} \mathbf{2}(\mathrm{C})$ and $\mathbf{H 3}$ (D). 
Table 3. AutoDock results in COX-2 for naproxen and the three hydrogelators.

\begin{tabular}{lcccc}
\hline \multicolumn{1}{c}{ Energy (kcal/mol) } & Naproxen & (H1) & (H2) & (H3) \\
\hline Estimated free energy of binding & -8.25 & -9.29 & -10.7 & -3.37 \\
Final intermolecular energy & -9.15 & -12.3 & -14.0 & -11.1 \\
Final total internal energy & -0.31 & -2.07 & -2.09 & -8.19 \\
Loss of torsional free energy & +0.89 & +2.98 & +3.28 & +7.76 \\
Unbound system energy & -0.31 & -2.07 & -2.09 & -8.19 \\
\hline
\end{tabular}

The anti-inflammatory effect will inhibit the immune system response to the presence of the hydrogel and the magnetic nanoparticles. Otherwise, the nanocarriers and nanoparticles could be phagocytised, trigger fever and/or be rejected by the organism.

Manganese ferrite nanoparticles. The X-ray diffraction (XRD) pattern of manganese ferrite nanoparticles (NPs) presents well defined peaks (Fig. 3), suggesting a structure of crystalline nature, even without calcination, as previously reported. ${ }^{2}$ Distinctive diffraction peaks are observed at $2 \theta=18.1^{\circ}, 29.8^{\circ}, 35.1^{\circ}, 36.7^{\circ}$, $42.7^{\circ}, 52.9^{\circ}, 56.3^{\circ}$ and $61.8^{\circ}$, which correspond to the (111), (220), (311), (222), (400), (422), (333), (511) and (440) plans of the $\mathrm{MnFe}_{2} \mathrm{O}_{4}$ crystalline structure, respectively. Rietveld analysis was carried out using a background defined by linear interpolation between a set of points at constant scattering angles and refinable intensities, resulting in a good fit, with $R_{f}$ and $\chi^{2}$ parameters of 6.53 and 1.77, respectively. A lower limit size estimation of $18.6 \mathrm{~nm}$ was obtained by the Scherrer equation, in agreement with the previously reported. ${ }^{2}$ The UV-Visible absorption spectrum of $\mathrm{MnFe}_{2} \mathrm{O}_{4}$ NPS (Figure S1 in Supplementary Information) is characteristic of an indirect semiconductor, as reported earlier. ${ }^{2,47}$ The long range of NPs absorption points to the possibility of a quenching effect of the emission of fluorescent drugs/probes incorporated in the magnetogel matrix, as previously observed in drug-loaded liposomes entrapping manganese ferrite nanoparticles. ${ }^{2,27}$ The magnetic properties of $\mathrm{MnFe}_{2} \mathrm{O}_{4}$ nanoparticles prepared by the same procedure were already described and a superparamagnetic behaviour for these nanoparticles was reported. ${ }^{2}$

Magnetogels containing $\mathrm{MnFe}_{2} \mathrm{O}_{4}$ nanoparticles. The intrinsic fluorescence of the naproxen moiety was explored to study the incorporation of nanoparticles in the hydrogel network. The hydrogels fluorescence spectra are dominated by the emission of the naproxen moiety, characterized by a non-structured band with maximum emission at $360 \mathrm{~nm}$ (Fig. 4). An additional band with maximum around $440-450 \mathrm{~nm}$ is associated with the emission of aggregates, as it was previously described. ${ }^{19}$

The hydrogel $\mathbf{H} \mathbf{3}$ self-assembly is characterized by a bathochromic shift with increasing concentration and a subtle increase in 430 $\mathrm{nm} .^{24}$ The absence of a strong aggregate emission band may be associated with the salt-bridged stabilized peptide backbone hampering naproxen moieties mutual approximation, while the higher hydrophobicity of $\mathbf{H} \mathbf{1}$ and $\mathbf{H} \mathbf{2}$ favours Npx moieties proximity, leading to a larger aggregation.

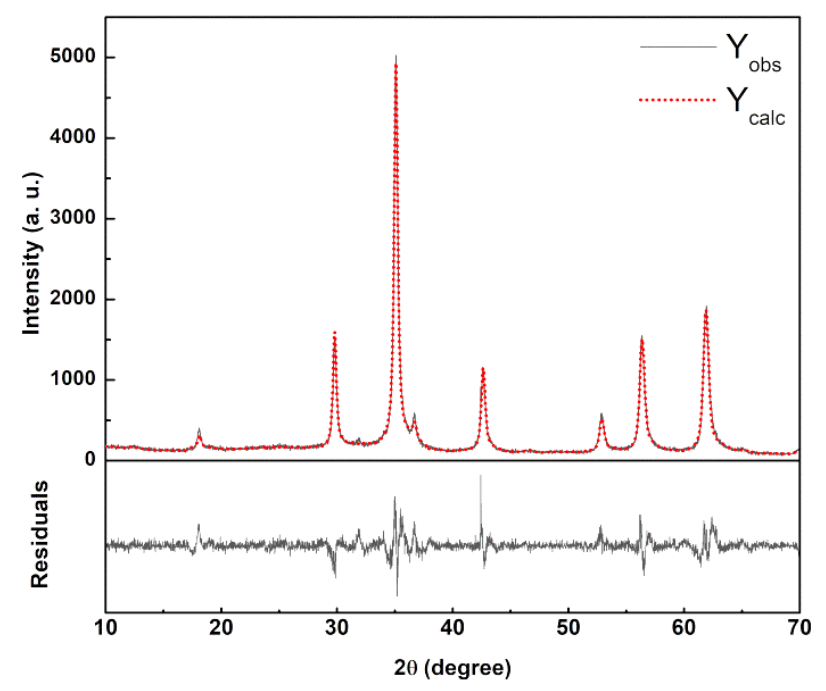

Fig. 3 XRD pattern of the $\mathrm{MnFe}_{2} \mathrm{O}_{4}$ nanoparticles.

The incorporation of magnetic nanoparticles results in quenching of hydrogels fluorescence, as it can be observed by comparison of magnetogels and hydrogels emission spectra (Fig. 4). This quenching effect may result from electronic energy transfer to nanoparticles, as they absorb in a wide energy range (Fig. S1).

The heavy atom effect can also contribute to fluorescence inhibition, considering that the presence of this kind of atoms (e.g. manganese) enhances spin-orbit coupling and, thus, the efficiency intersystem crossing process. ${ }^{43}$

Apart from quenching, the emission features of the magnetogels are very similar to the ones of plain hydrogels (Fig. 4), showing that manganese ferrite nanoparticles have no significant influence in hydrogel fibres assembly, that have already proven to be suitable for carrying the potential anticancer drugs tested here in magnetogels. ${ }^{19,24}$

Still, a more pronounced quenching effect is observed for the RGD containing hydrogel $(\mathbf{H} \mathbf{3})$, which can be an indication that the nanoparticles are closer to the emissive aromatic groups in this hydrogel matrix. Such might be due to stronger interactions between the nanoparticles and the hydrogelator polar and ionic groups when compared to the other hydrogelators, as $\mathbf{H} \mathbf{3}$ presents more groups available for coordination of the nanoparticles metal ions and hydrogen bonding with $\mathrm{OH}^{-}$groups. 


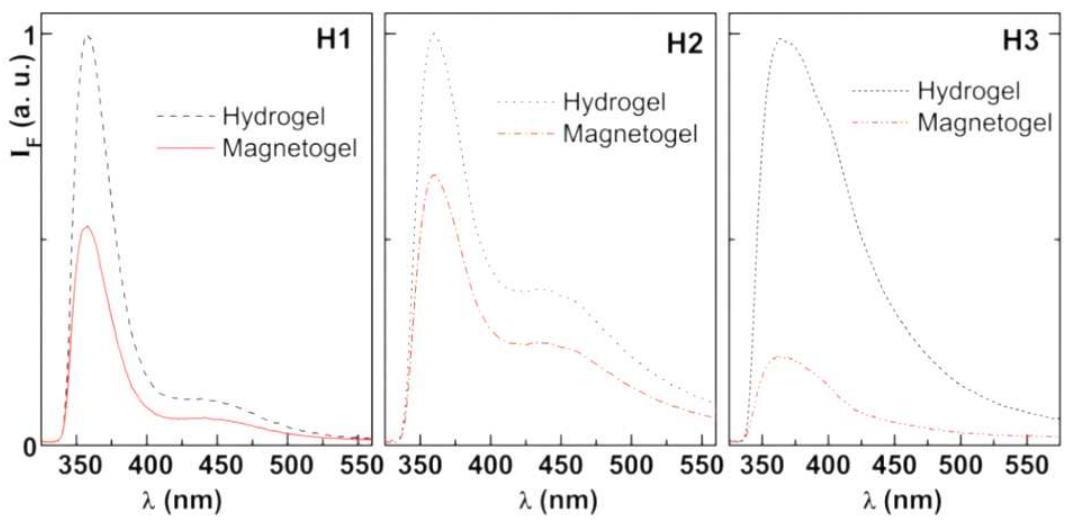

Fig. 4 Fluorescence spectra $\left(\lambda_{\text {exc }}=290 \mathrm{~nm}\right)$ of hydrogels and corresponding magnetogels containing manganese ferrite nanoparticles. H1: Npx-L-Phe-Z- $\triangle$ Abu-OH; H2: Npx-L-Trp-Z- $\Delta$ Phe-OH; H3: Npx-L-Ala-Z- $\Delta$ Phe-Gly-L-Arg-Gly-L-Asp-Gly-OH.

The three-dimensional structures of the magnetogels obtained by TEM are presented in Figure 5. All the magnetogels show randomly distributed fibrous networks with a noticeable heterogeneity in the dimensions of the fibres. The magnetogel $\mathbf{H} \mathbf{1}$ exhibits fibres with 20.(3) \pm 5 .(8) $\mathrm{nm}$ (SD, standard deviation) of width, where the thickest and thinnest fibre width determined as $31.1 \mathrm{~nm}$ and $10.9 \mathrm{~nm}$, respectively. The magnetogel $\mathbf{H 2}$ also shows fibre diameters close to those obtained for the previous magnetogel, with a mean value of $20 .(4) \pm 4$.(1) $\mathrm{nm}$ (SD), the largest being $32.8 \mathrm{~nm}$ and the thinnest down to $14.2 \mathrm{~nm}$. The magnetogel $\mathrm{H3}$ exhibits some fibres with a mean diameter of 6.(3) \pm 1 . (8) $\mathrm{nm}$ (SD), the largest being $8.6 \mathrm{~nm}$ and the thinnest $3.2 \mathrm{~nm}$. Interestingly, the magnetogel fibres exhibit some differences in size when compared to the neat hydrogel samples (without magnetic nanoparticles) previously reported, ${ }^{19,23}$ where the hydrogel $\mathbf{H 1}$ presented fibres of $10 \mathrm{~nm},{ }^{23}$ while $\mathbf{H} \mathbf{2}$ was far larger, attaining dimensions from 44 to $260 \mathrm{~nm}$, depending on the density of the network. ${ }^{19}$ Figure 5D evidences that the magnetogel $\mathbf{H} \mathbf{2}$ has fibre diameters around $\mathbf{3 0}$ $\mathrm{nm}$, where a nanoparticle with $c a .20 \mathrm{~nm}$ diameter can be observed adsorbed to the fibres surface. Moreover, the magnetic nanoparticles show a mean diameter of 23.(9) \pm 2 .(9) $\mathrm{nm}(\mathrm{SD})$, in close agreement with the size determined from XRD. TEM images of $\mathrm{H3}$ magnetogel (Fig. 5E and 5F) were obtained without contrast agent, as it does not allow a proper differentiation of fibres and nanoparticles. As verified in fluorescence measurements (Fig. 4) and FTIR assays, TEM images also reveal that the hydrogel strongly binds to the nanoparticles, forming an organic coating and stablishing cross-links between the nanoparticles. In fact, the magnetogel shows a different architecture from the hydrogel, justifying the stronger emission quenching owing to the proximity of the chromophore Npx to the nanoparticles.

It must be emphasized that no nanoparticles aggregation is observed during magnetogels preparation. Moreover, more than $90 \%$ of the nanoparticles remain encapsulated during gel formation (2-3 h), as inferred from UV-Visible absorption measurements (Figure S2 in Supplementary Information). Measured zeta potential values were $-15.0 \mathrm{mV}$ for magnetic nanoparticles dispersed in water, $-6.8 \mathrm{mV}$ for magnetogel $\mathbf{H 1}$ and -11.7 $\mathrm{mV}$ for magnetogel $\mathbf{H 2}$.
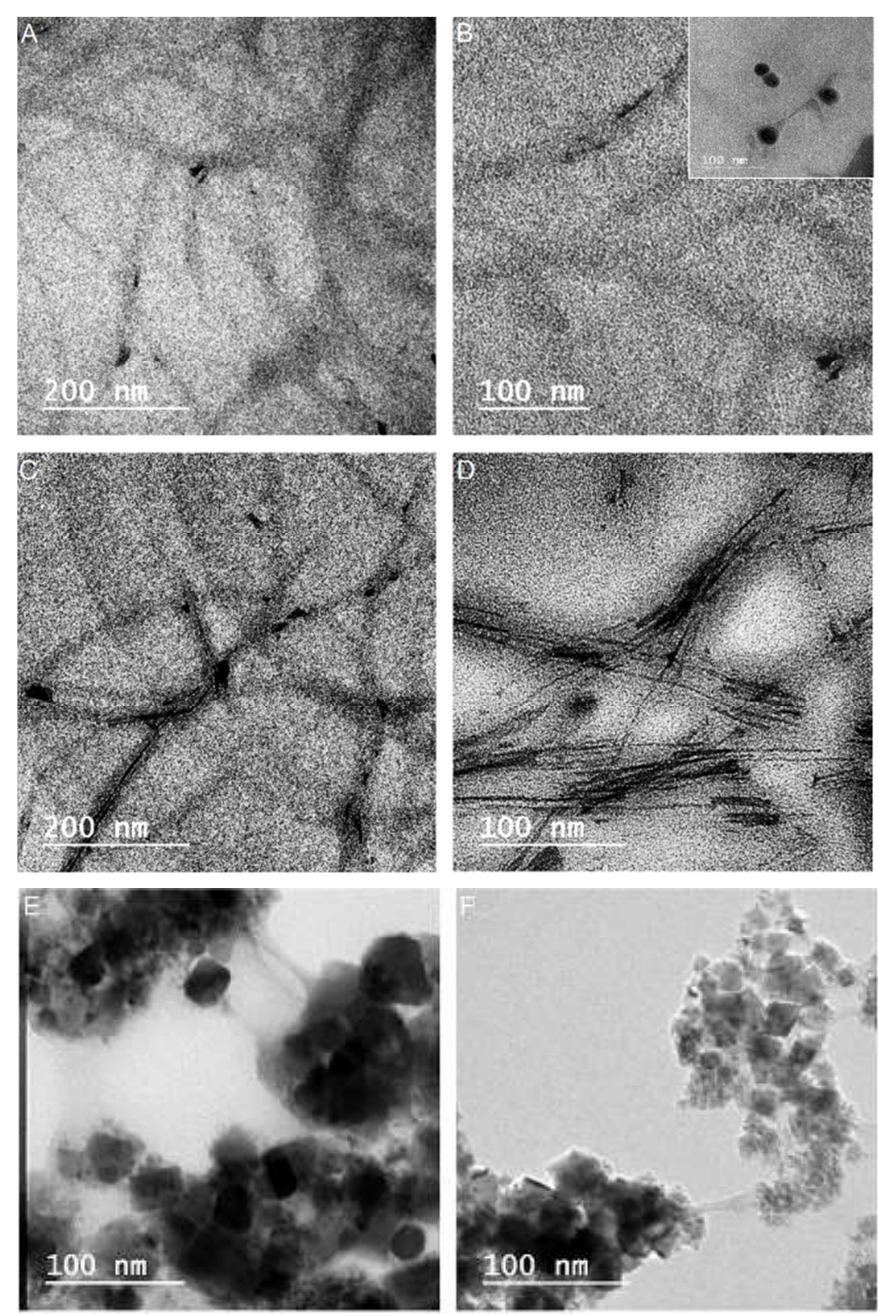

Fig. 5 Transmission Electron Microscopy images of the magnetogels based on $\mathbf{H} \mathbf{1}$ ( $\mathbf{A}$ and $\mathbf{B}), \mathbf{H} \mathbf{2}$ (C and $\mathbf{D}$ ) and $\mathbf{H} \mathbf{3}$ (E and $\mathbf{F}$ ). Inset in image $\mathbf{B}$ : Manganese ferrite nanoparticles observed in the magnetogel $\mathbf{H} \mathbf{1}$.

The effect of incorporation of magnetic nanoparticles in the self-assembled dehydropeptide hydrogels was also investigated by FTIR measurements. Figure 6 displays FTIR spectra of the 
magnetogels and the corresponding ones for the neat hydrogels. It can be observed that IR spectra of hydrogels and magnetogels do not exhibit any significant differences in the vibrational frequencies. The major difference is that the IR spectra are more intense for magnetogels, besides being generally sharper in the $3000-3500 \mathrm{~cm}^{-1}$ region. This rise in intensity could be related to an increase of dipolar moments variation induced by the $\mathrm{MnFe}_{2} \mathrm{O}_{4}$ nanoparticles, while differences in intensity ratios indicate that structural or physicochemical changes occur in the presence of nanoparticles. Wavenumbers were assigned according to previous studies involving naproxen and free amino acids ${ }^{48,49}$ (Table S1 in Supplementary Information).

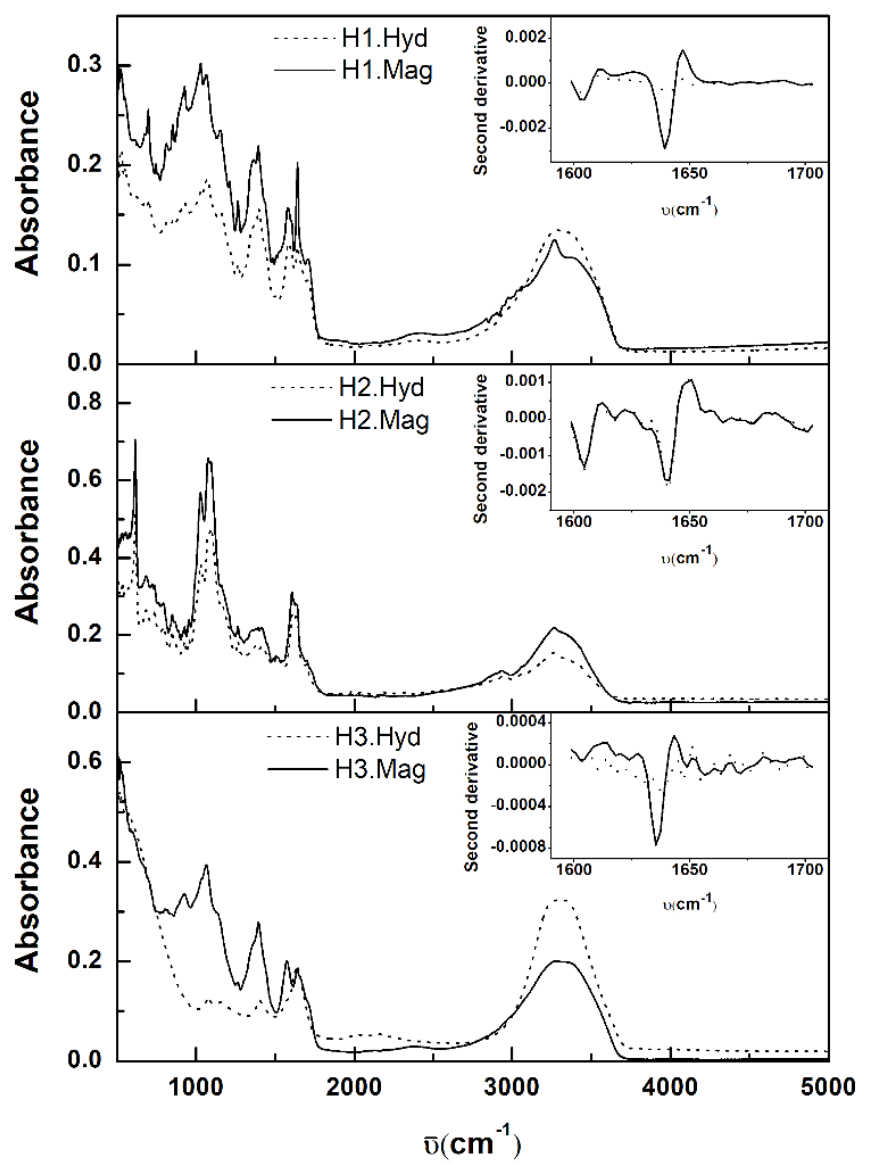

Fig. 6 Infrared spectra of hydrogels (Hyd) and corresponding magnetogels (Mag) containing manganese ferrite nanoparticles, in the $500-5000 \mathrm{~cm}^{-1}$ region. Inset: Second derivative of amide I region of the respective hydrogel and magnetogel.

The RGD magnetogel $\mathbf{H 3}$ exhibits some vibrational changes, namely, the $1160 \mathrm{~cm}^{-1}$ peak shifts to $1140 \mathrm{~cm}^{-1}$, which is assigned to $\mathrm{NH}_{3}^{+}$ rocking of arginine side chain; and the $1079 \mathrm{~cm}^{-1}$ shifts to $1066 \mathrm{~cm}^{-1}$, which can be identified as the $\mathrm{C}-\mathrm{N}$ vibration, suggesting that some interactions might have taken place between the arginine side chain and the magnetic nanoparticles, thus supporting the stronger quenching previously observed in fluorescence assays.

Considering that amide I band gives information over the secondary structure of peptides, the second-derivative was used to identify overlapping bands, assignments being performed according to the reported values. ${ }^{50-52}$
The hydrogels exhibit a peak close to $1616 \mathrm{~cm}^{-1}$ associated to the presence of $\beta$-strands in aggregated structures, showing other minor peaks characteristic of $\beta$-sheets in the range $1620-1630 \mathrm{~cm}^{-1}$, which reflect changes in hydrogen bonding or transition dipole couplings, ${ }^{51}$ the sharpest peak arising at $1641 \mathrm{~cm}^{-1}$. Other minor peaks are found around $1680 \mathrm{~cm}^{-1}$, which can be a result of strong hydrogen bonding between $\beta$-sheets or due to turns, ${ }^{51}$ and at $1697 \mathrm{~cm}^{-1}$, evidencing $\beta$-strand aggregates.

Comparison of $\mathbf{H} \mathbf{1}$ hydrogel and magnetogel shows that the main difference relies on the fact that the magnetogel has a strong band with peak at $1639 \mathrm{~cm}^{-1}$, suggesting that, in the presence of magnetic nanoparticles, the hydrogelator molecules are more randomly oriented. However, the amide I band second derivative of $\mathbf{H} \mathbf{2}$ hydrogel and magnetogel displays no vibrational changes between each other, suggesting that both converged to similar final secondary structures. The peaks around $1656 \mathrm{~cm}^{-1}, 1664 \mathrm{~cm}^{-1}$, and $1675 \mathrm{~cm}^{-1}$ are assigned to random coil, loop and turn structures, respectively. Minor peaks are also observed at $1691 \mathrm{~cm}^{-1}$ and $1697 \mathrm{~cm}^{-1}$ associated to $\beta$-strands in aggregated structures. Finally, regarding $\mathbf{H 3}$, the magnetogel exhibits an intense peak at $1635 \mathrm{~cm}^{-1}$ not observed for the hydrogel (the latter possessing instead two peaks at $1629 \mathrm{~cm}^{-1}$ and $1637 \mathrm{~cm}^{-1}$ ), suggesting that the nanoparticles also enhanced the hydrogelator self-assembly into $\beta$-sheet structures.

To obtain a quantitative insight on the contribution of secondary structures for the gelation process, a curve-fitting approach was used, where peaks were fitted according to the higher resolution provided by the second derivative (Table 4). In general, the nanoparticles produce some conformational changes in all hydrogels, with a reduction of ordered secondary structures in the case of $\mathbf{H} \mathbf{1}$ and $\mathbf{H} \mathbf{3}$ and a small influence on $\mathbf{H 2}$, where the decrease of random coils is accompanied by the increase of turns and loops. Interestingly, even if the arginine residue contributes around 1633 $\mathrm{cm}^{-1}$ and $1673 \mathrm{~cm}^{-1}$, the $\mathbf{H} \mathbf{3}$ magnetogel still shows a reduction of $\beta$ sheets compared to the hydrogel, accompanied by the increasing unordered structures also observed in the other magnetogels. Considering that a higher unordered structures percentage might reduce the compactness of the well-ordered self-assembled structures owing to the lack of ordered hydrogen bonding, the magnetogels are expected to have thicker fibres than the hydrogels. The amide I band analysis presents some limitations, such as the water interference, that highly affects the secondary structure percentages and the ambiguity in the assignment of random coil and $\alpha$-helix bands (owing to their overlap). On the other hand, the amide III region is not contaminated by the water signal, apart from a better localization of the different amide bond vibrations contributions, the major drawback being the interference of other vibrational groups and the lack of distinction between random coil and turn frequencies. Nevertheless, although the analysis using amide I suggests a higher percentage of $\beta$-sheets (may be due to water contamination), when compared to amide III (Table 4), both results demonstrate that the incorporation of magnetic nanoparticles contributes to a reduction of $\beta$-sheet structures, that occurs at the expense of the increasing number of unordered structures. 
Table 4. Percentage of secondary structures in hydrogels and magnetogels, according to the general range reported in literature, ${ }^{51-53}$ obtained through curve-fitting of the amide I and amide III region.

\begin{tabular}{|c|c|c|c|c|c|c|c|}
\hline & \multirow{2}{*}{ Secondary structure } & \multicolumn{2}{|c|}{ H1 } & \multicolumn{2}{|c|}{$\mathrm{H} 2$} & \multicolumn{2}{|c|}{ H3 } \\
\hline & & Hydrogel & Magnetogel & Hydrogel & Magnetogel & Hydrogel & Magnetogel \\
\hline \multirow{4}{*}{ Amide I } & $\beta$-sheet & 54.11 & 33.12 & 64.53 & 63.17 & 58.84 & 49.8 \\
\hline & Random coil & 25.47 & 44.96 & 22.14 & 6.84 & 30.22 & 19.05 \\
\hline & $\alpha$-helix & 17.56 & 11.16 & 3.50 & 2.20 & 2.86 & 10.22 \\
\hline & Turns and loops & 2.86 & 10.75 & 9.83 & 27.78 & 8.09 & 20.93 \\
\hline \multirow{4}{*}{ Amide III } & $\beta$-sheet & 28.29 & 26.70 & 27.45 & 23.40 & 54.11 & 33.56 \\
\hline & Random coil & 58.29 & 50.40 & 48.49 & 41.34 & 10.86 & 46.23 \\
\hline & $\alpha$-helix & 5.60 & 5.60 & 2.41 & 2.056 & 22.26 & 10.97 \\
\hline & Turns and loops & 7.82 & 17.30 & 21.64 & 33.20 & 12.77 & 9.24 \\
\hline
\end{tabular}

Incorporation of potential antitumor drugs in the matrices of magnetogels. It was already reported that the hydrogel $\mathbf{H 2}$ is a potential drug nanocarrier, ${ }^{19}$ while the RGD-containing hydrogel (H3) has shown to be suitable for drug delivery applications using curcumin. $^{24}$ Here, this concept is further explored for the magnetogels containing manganese ferrite NPs. For that purpose, both the antitumor thienopyridine derivative and curcumin were incorporated in the three magnetogels matrices. Taking advantage of fluorescence properties of both hydrogels and drugs, a FRET (Förster Resonance Energy Transfer) process from the hydrogel emissive moieties (as the energy donors) to the drugs (energy acceptors) can be useful to investigate drug location, as the spectral overlap between drug absorption and hydrogel fluorescence is significant in both cases (Fig. S3 and S4 in Supplementary information). Another feature to take into account is that neither the thienopyridine derivative nor curcumin are emissive in aqueous media, ${ }^{24,29}$ both molecules possessing a hydrophobic nature.

Figure 7 displays the fluorescence spectra of the three magnetogels incorporating the antitumor thienopyridine derivative, exciting the hydrogelator naproxen moiety (Fig. 7A) and exciting only the antitumor drug (Fig. 7B). For comparison, the spectra of magnetogels without compound are also shown in Fig. 7A. Exciting the naproxen moiety of the hydrogelator molecules, an energy transfer can be observed from the hydrogel to the compound, evidenced by a quenching of the naproxen emission and an increase in the region of compound fluorescence $\left(\lambda_{\max } \sim 450 \mathrm{~nm}\right){ }^{29}$ It should, however, be noted that the antitumor compound is also directly excited at this wavelength $\left(\lambda_{\text {exc }}=290 \mathrm{~nm}\right)$. The increase in emission at the compound region is only tenuous in the magnetogel with RGD sequence (H3), which can be due to a more hydrophilic environment of the compound promoted by the RGD moiety or a higher quenching effect of drug (FRET acceptor) emission by the nanoparticles in this magnetogel. Nevertheless, a strong fluorescence emission is observed exciting only the antitumor compound (Fig. 7B) in all magnetogels, proving the incorporation of this drug in the gels matrix, as it is not fluorescent in aqueous media. $^{29}$

A similar investigation was performed for the model drug curcumin in the three magnetogel matrices. It can be observed (Fig. 8) a FRET occurrence, but with some notable differences. Regarding the magnetogel $\mathbf{H 1}$, a decrease in the donor (hydrogel) emission band is detected in the gel incorporating curcumin, but the band corresponding to drug emission is not clearly observed. Even so, a higher ratio between the emission bands at $440 \mathrm{~nm}$ and $360 \mathrm{~nm}$ is inferred (Fig. 8A). Still, when exciting curcumin alone, its fluorescence appears with a maximum around $500 \mathrm{~nm}$ (Fig. 8B), indicating a slightly polar environment, similar to the one felt by this drug in chloroform. ${ }^{24}$ The occurrence of FRET and corresponding increase of curcumin emission is more clearly observed in magnetogel $\mathbf{H} \mathbf{2}$. The fluorescence band of the drug shows that it reports a more polar environment when incorporated in magnetogels $\mathbf{H} \mathbf{2}$ and $\mathbf{H} \mathbf{3}\left(\lambda_{\max }\right.$ at $\left.c a .525-530 \mathrm{~nm}\right)$, similar to acetonitrile, as previously reported for this drug in $\mathbf{H 3}$ hydrogel (without magnetic nanoparticles). ${ }^{24}$

Table 5 displays the donor-acceptor distances between the gel emissive moieties and the drugs, calculated from the FRET process. It can be observed that, in the case of thienopyridine incorporated in magnetogel $\mathbf{H 2}$, the donor-acceptor distance is slightly smaller than the one observed for the same drug in the corresponding hydrogel $(2.3 \mathrm{~nm}){ }^{19}$ indicating that the presence of the magnetic nanoparticles may influence the drug location in the gel matrix. Regarding curcumin, previous studies in the RGD containing hydrogel (H3) (0.4 wt\%) allowed determining a donor-acceptor distance of $2.61 \mathrm{~nm},{ }^{24}$ very similar to the one in the corresponding magnetogel (Table 5). Nevertheless, a host/guest-type interaction (magnetogel being the host and drug the guest) is anticipated in all cases, considering that the determined donor-acceptor distances are similar to the ones reported in literature for the non-covalent binding of these drugs with serum albumin (human, HSA, or bovine, $\mathrm{BSA}$ ), around $2.5 \mathrm{~nm}$ for the thienopyridine derivative in BSA (at $\mathrm{pH}=7),{ }^{29}$ and $3.1 \mathrm{~nm}$ for curcumin interacting with $\mathrm{HSA}^{54}$

Fluorescence (steady-state) anisotropy measurements were performed to assess the rotational mobility of the drugs when incorporated in the magnetogels (Table 6). The higher the degree of rotation of the fluorescent drug, the lower is the anisotropy, as the latter is proportional to the rotational correlation time of the fluorescent moiety. 
Table 5. FRET efficiencies $\left(\Phi_{\text {FRET }}\right)$, fluorescence quantum yields of the donor $\left(\Phi_{D}^{\mathbf{0}}\right)$ and donor-acceptor distances $\left(R_{\mathrm{DA}}\right)$ for magnetogels with incorporated antitumor drugs.

\begin{tabular}{|c|c|c|c|c|}
\hline Drug & Magnetogel & $\Phi_{\text {FRET }}$ & $\Phi_{D}^{0}{ }^{a}$ & $R_{\mathrm{DA}}(\mathrm{nm})$ \\
\hline \multirow{3}{*}{$\begin{array}{c}\text { Thienopyridine } \\
\text { derivative }\end{array}$} & Npx-L-Phe-Z- $\triangle$ Abu-OH (H1) & 0.24 & 0.021 & 2.1 \\
\hline & Npx-L-Trp-Z- $\Delta \mathrm{Phe}-\mathrm{OH}(\mathbf{H} \mathbf{2})$ & 0.40 & 0.009 & 2.0 \\
\hline & Npx-L-Ala-Z- $\Delta$ Phe-Gly-L-Arg-Gly-L-Asp-Gly-OH (H3) & 0.38 & 0.010 & 2.1 \\
\hline \multirow{3}{*}{ Curcumin } & Npx-L-Phe-Z- $\triangle$ Abu-OH (H1) & 0.35 & 0.021 & 2.6 \\
\hline & Npx-L-Trp-Z- $\Delta$ Phe-OH (H2) & 0.19 & 0.009 & 2.2 \\
\hline & Npx-L-Ala-Z- $\Delta$ Phe-Gly-L-Arg-Gly-L-Asp-Gly-OH (H3) & 0.38 & 0.010 & 2.5 \\
\hline
\end{tabular}

${ }^{a}$ Relative to $L$-Tryptophan in aqueous buffer solution, $\mathrm{pH}=7.2\left(\Phi_{\mathrm{r}}=0.14\right.$ at $\left.25^{\circ} \mathrm{C}\right) .{ }^{42}$ Error about $10 \%$.

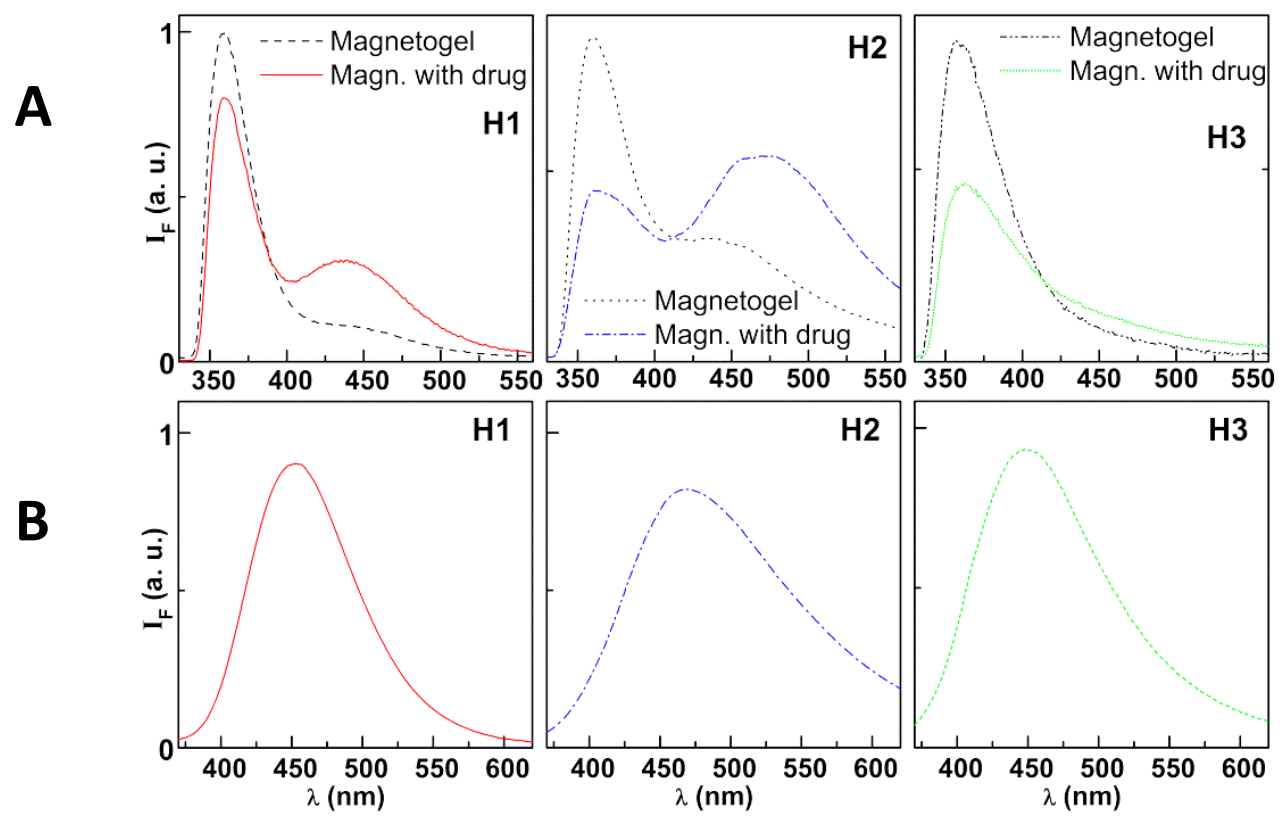

Fig. 7 A: Fluorescence spectra $\left(\lambda_{\text {exc }}=290 \mathrm{~nm}\right)$ of magnetogels incorporating the thienopyridine derivative and comparison with plain magnetogels. B: Fluorescence spectra $\left(\lambda_{\text {exc }}=360 \mathrm{~nm}\right)$ of magnetogels exciting only the thienopyridine derivative.

H1: Npx-L-Phe-Z- $\triangle A$ Abu-OH; H2: Npx-L-Trp-Z- $\triangle$ Phe-OH; H3: Npx-L-Ala-Z- $\Delta$ Phe-Gly-L-Arg-Gly-L-Asp-Gly-OH.

Table 6. Steady-state fluorescence anisotropy $(r)$ values for the thienopyridine derivative and curcumin in the several magnetogels.

\begin{tabular}{ccc}
\hline Drug & Magnetogel & $\boldsymbol{r}^{a}$ \\
\hline \multirow{2}{*}{ Thienopyridine } & H1 & 0.017 \\
derivative & H2 & 0.129 \\
& H3 & 0.107 \\
\hline \multirow{3}{*}{ Curcumin } & H1 & 0.266 \\
& H2 & 0.128 \\
& H3 & 0.245 \\
\hline
\end{tabular}

${ }^{a}$ Anisotropy values of drugs in glycerol at $25^{\circ} \mathrm{C}$ :

Thienopyridine derivative: $r=0.327 .^{29}$

Curcumin: $r=0.365 .^{24}$
It can be observed that the thienopyridine derivative seems to have a special affinity for the magnetogel of the hydrogelator $\mathbf{H} \mathbf{2}$, where the distance to the aromatic moieties of the hydrogel is smaller and the rotation of the drug is more hindered (Table 6). In magnetogel H1, the low value of $r$ would indicate that this drug is in a fluid environment, rotating almost freely. But the emission maximum of the thienopyridine derivative near $460 \mathrm{~nm}$, similar to cyclohexane, ${ }^{29}$ points to a non-polar environment, corresponding to the interior of the gel fibres. This location would originate a high anisotropy, as observed for curcumin in this magnetogel. A possible explanation for the observed low anisotropy is that a significant part of the emission from thienopyridine originates from excitation of hydrogel aggregates, followed by energy transfer to the drug. This is possible as the excitation spectra of hydrogel $\mathbf{H} \mathbf{1}$ in the aggregate region $\left(\lambda_{\mathrm{em}}=450 \mathrm{~nm}\right.$ ) extends above $350 \mathrm{~nm}^{23}$ (Fig. S5 in Supplementary 
Information), showing that aggregates can be excited at $360 \mathrm{~nm}$. As this low anisotropy value does not occur for curcumin, energy transfer from $\mathbf{H} \mathbf{1}$ aggregates is not efficient, indicating that the location of curcumin (although also on the fibres) is different from that of the thienopyridine derivative.

Assuming a similar excited-state lifetime for each drug in the three magnetogels, it can be inferred that curcumin rotation is more hindered in magnetogels derived from hydrogelators $\mathbf{H} \mathbf{1}$ and $\mathbf{H} \mathbf{3}$. Overall, it can be concluded that both drugs interact with magnetogels by a host/guest type interaction and that the three magnetogels are suitable carriers either for curcumin or the thienopyridine derivative, as it was previously anticipated for the hydrogel $\mathbf{H} \mathbf{2}$ (incorporating the thienopyridine compound) ${ }^{19}$ and the RGD-containing hydrogel H3 for curcumin. ${ }^{24}$
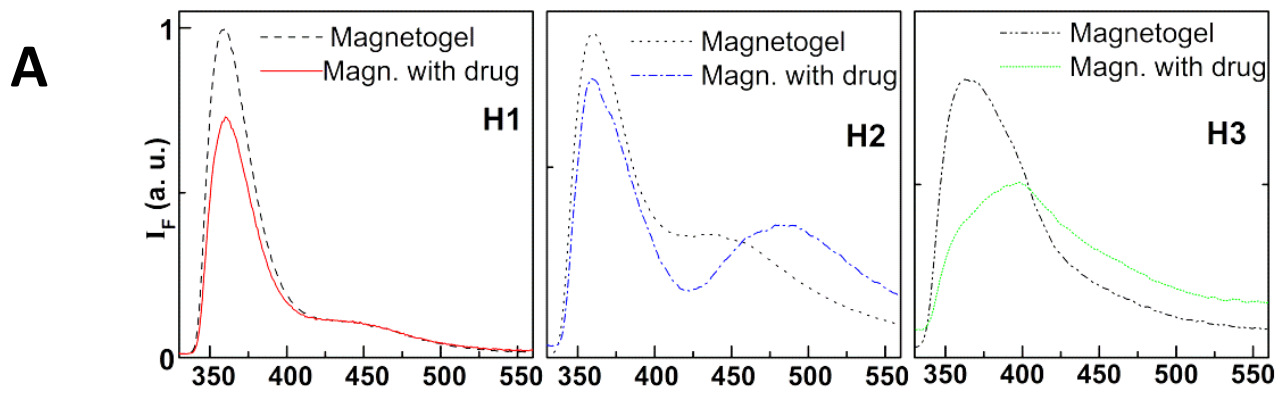

B
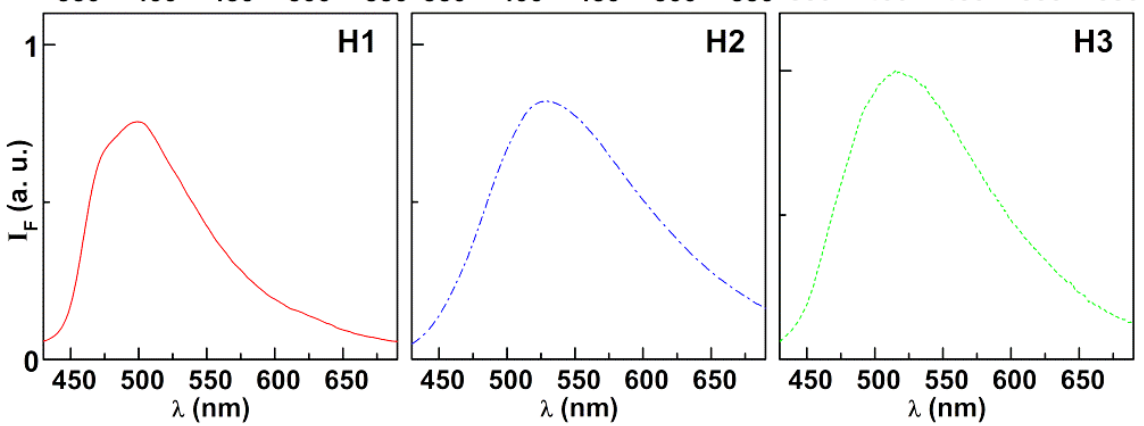

Fig. 8 A: Fluorescence spectra $\left(\lambda_{\text {exc }}=290 \mathrm{~nm}\right)$ of magnetogels incorporating curcumin and comparison with plain magnetogels.

B: Fluorescence spectra ( $\lambda_{\text {exc }}=410 \mathrm{~nm}$ ) of magnetogels exciting only curcumin.

H1: Npx-L-Phe-Z- $\triangle$ Abu-OH; H2: Npx-L-Trp-Z- $\triangle$ Phe-OH; H3: Npx-L-Ala-Z- $\Delta$ Phe-Gly-L-Arg-Gly-L-Asp-Gly-OH.

Drug transport towards model membranes (SUVs). In order to assess the potential of these magnetogels to release drugs into cell membranes, small unilamellar vesicles (SUVs) composed of $70 \%$ phosphatidylcholine (PC) and $30 \%$ cholesterol (Ch) were prepared. Mean hydrodynamic diameter of these SUVs was determined by DLS as $92.8 \pm 3.1 \mathrm{~nm}$, in accordance to those previously determined for similar systems. ${ }^{55}$ These PC:Ch (7:3) vesicles are often used as membrane models in studies of drug permeation. ${ }^{56,57}$ The lipid probe Nile $\operatorname{Red}^{37-39}$ was incorporated in these vesicles to monitor drug release from magnetogels into the SUVs membrane. For that purpose, FRET assays using the potential drugs as energy donors and Nile Red as the acceptor were carried out, once antitumor drug/Nile Red are favourable donor-acceptor pairs, especially in the case of curcumin (Figure S6 in Supplementary Information). After drug-loaded magnetogels were left in contact for 2 hrs with SUVs containing Nile Red, an energy transfer from the antitumor drug (thienopyridine derivative or curcumin) to Nile Red was detected, exciting only the drug (Fig. 9).

For the hydrogels $\mathbf{H} \mathbf{1}$ and $\mathbf{H} \mathbf{2}$ loaded with the thienopyridine derivative, after interaction with SUVs, while the decrease in drug emission is detected, the increase in the Nile Red fluorescence is extremely tenuous (Fig. 9A). For the magnetogel possessing RGD, the FRET process is clearly more visible. For the curcumin-loaded magnetogels, Nile Red emission is detected in all cases upon curcumin excitation (Fig. 9B). Furthermore, a shift in the curcumin emission band to lower wavelengths (from 525-530 nm to $c a .500$ $\mathrm{nm}$ ) is detected after interaction in the case of magnetogels $\mathbf{H} \mathbf{2}$ and H3 (in H1, curcumin band was already near $500 \mathrm{~nm}$ in the magnetogel). This represents a clear proof that curcumin is released in the membrane of lipid vesicles containing Nile Red, feeling a more hydrophobic environment in these membranes.

Donor-acceptor (drug-Nile Red) distances were calculated from equations (3) to (6). The fluorescence quantum yield, $\Phi_{\boldsymbol{D}}^{\mathbf{0}}$, of each donor (thienopyridine derivative or curcumin) in these lipid vesicles was determined by the standard method (eqn. (1)) (Table 7). FRET efficiencies are higher in the case of curcumin, corresponding to generally lower donor-acceptor (drug-Nile Red) distances in SUVs. A cause for the lower values of $\Phi_{\text {FRET }}$ for the thienopyridine derivative is the lower spectral overlap with Nile Red (Figure S6A in Supplementary Information).

In magnetogel $\mathbf{H 1}$ (Fig. 9A), a similar microenvironment in both hydrogel matrix and liposomes will produce a lower net force leading to the transport of the drug from $\mathbf{H} \mathbf{1}$ towards SUVs than in the other magnetogels. Such similar microenvironment is also suggested by the absence of shift in curcumin emission after interaction of this magnetogel with SUVs. 


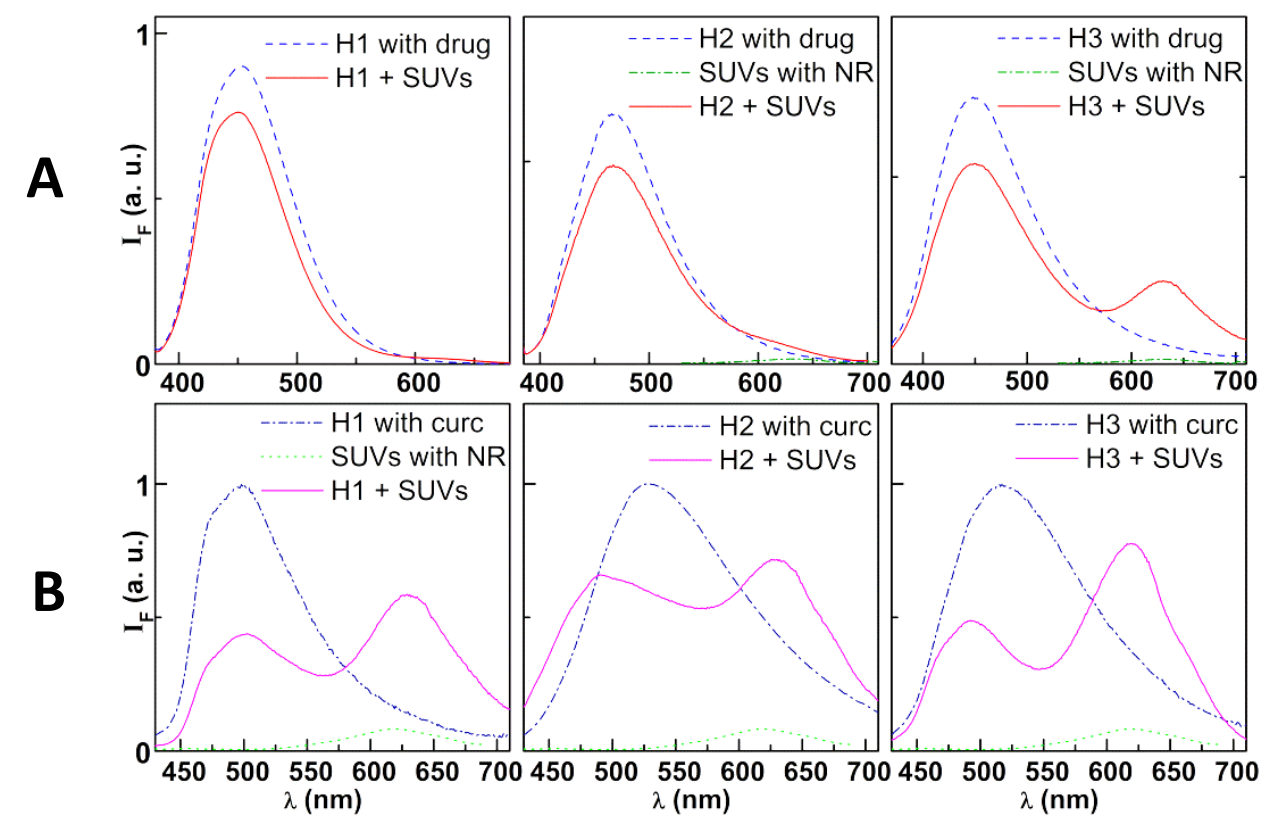

Fig. 9 Fluorescence emission spectra of drug-loaded magnetogels, SUVs loaded with Nile Red (at drug excitation wavelength) and after interaction of drug-loaded magnetogels with SUVs. A: Magnetogels loaded with antitumor thienopyridine derivative $\left(\lambda_{\text {exc }}=360 \mathrm{~nm}\right) ; \mathbf{B}$ : Magnetogels loaded with curcumin $\left(\lambda_{\text {exc }}=420 \mathrm{~nm}\right)$.

H1: Npx-L-Phe-Z- $\triangle$ Abu-OH; H2: Npx-L-Trp-Z- $\Delta$ Phe-OH; H3: Npx-L-Ala-Z- $\Delta$ Phe-Gly-L-Arg-Gly-L-Asp-Gly-OH.

Table 7. FRET efficiencies $\left(\Phi_{\text {FRET }}\right)$ and donor-acceptor distances $\left(R_{\mathrm{DA}}\right)$ for the interaction of drug-loaded magnetogels and model membranes containing Nile Red.

\begin{tabular}{|c|c|c|c|c|}
\hline Drug & $\Phi_{D}^{0}$ & Magnetogel & $\Phi_{\text {FRET }}{ }^{c}$ & $R_{\mathrm{DA}}(\mathrm{nm})$ \\
\hline \multirow{3}{*}{$\begin{array}{c}\text { Thienopyridine } \\
\text { Derivative }\end{array}$} & \multirow{3}{*}{$0.057^{a}$} & Npx-L-Phe-Z- $\triangle$ Abu-OH (H1) & 0.18 & 2.8 \\
\hline & & Npx-L-Trp-Z- $\Delta$ Phe-OH (H2) & 0.17 & 3.1 \\
\hline & & Npx-L-Ala-Z- $\Delta$ Phe-Gly-L-Arg-Gly-L-Asp-Gly-OH (H3) & 0.25 & 2.8 \\
\hline \multirow{3}{*}{ Curcumin } & \multirow{3}{*}{$0.064^{b}$} & Npx-L-Phe-Z- $\triangle$ Abu-OH (H1) & 0.55 & 2.6 \\
\hline & & Npx-L-Trp-Z- $\Delta$ Phe-OH (H2) & 0.37 & 2.8 \\
\hline & & Npx-L-Ala-Z- $\Delta$ Phe-Gly-L-Arg-Gly-L-Asp-Gly-OH (H3) & 0.45 & 2.7 \\
\hline
\end{tabular}

${ }^{a}$ Relative to quinine sulfate in $0.05 \mathrm{M}$ sulfuric acid $\left(\Phi_{\mathrm{r}}=0.546\right.$ at $\left.25^{\circ} \mathrm{C}\right) .{ }^{58}$ Error about $10 \%$.

${ }^{b}$ Relative to fluorescein in $0.1 \mathrm{M} \mathrm{NaOH}\left(\Phi_{\mathrm{r}}=0.93\right.$ at $\left.25^{\circ} \mathrm{C}\right) .{ }^{59}$ Error about $10 \%$.

${ }^{c}$ The donor integrated fluorescence intensity in the absence of acceptor was determined in SUVs containing only curcumin.

Nevertheless, donor-acceptor distances in SUVs are generally higher in the case of the thienopyridine derivative. Considering both drugs, the magnetogels $\mathbf{H} \mathbf{1}$ and $\mathbf{H} \mathbf{3}$ seem to be more effective in performing drug release into SUVs membrane.

Nile Red is a lipid probe, well-known for its strong affinity to lipid membranes. These membranes have usually 7-9 nm thickness. ${ }^{60}$ Therefore, a distance of 2-3 $\mathrm{nm}$ between the drugs and Nile Red (FRET donor-acceptor distance in SUVs, Table 7) is a clear indication that these drugs cross and penetrate the SUVs membrane, being close to Nile Red. Moreover, after interaction with SUVs, curcumin emission (which is sensitive to the polarity of environment) is shifted to lower wavelengths, indicating that this drug is located in a more hydrophobic environment (the lipid membrane of SUVS).
Thermodynamics is the driving force for drug release into SUVs, as an equilibrium will be established between the drug in hydrogel hydrophobic cavities and the hydrophobic membrane of SUVs.

After $2 \mathrm{~h}$ of interaction of hydrogels with SUVs, no shift in Nile Red emission is observed. This is an evidence that the integrity of the SUVs is preserved, as the lipid probe Nile Red is very sensitive to the polarity of the environment (although the possibility of rupture cannot be excluded in a long term).

Overall, the results here obtained point to promising applications of these magnetogels to transport drugs more efficiently to cells and allowing their release by interaction with cell membranes. 


\section{Conclusions}

In this work, manganese ferrite nanoparticles were prepared and incorporated in the matrix of previously synthesized dehydropeptide-derived hydrogels containing naproxen, namely Npx-L-Phe-Z- $\triangle$ Abu-OH, Npx-L-Trp-Z- $\triangle$ Phe-OH and Npx-L-Ala-Z- $\Delta$ PheGly-L-Arg-Gly-L-Asp-Gly-OH.

The as-formed magnetogels were then tested as nanocarriers for potential antitumor drugs, a thienopyridine derivative and the natural compound curcumin. The intrinsic fluorescence of both drugs was used as a valuable tool for this investigation. Therefore, FRET assays (from the gels aromatic moieties to the drugs) and fluorescence anisotropy measurements were carried out and confirmed incorporation of both drugs in the magnetogels matrices. The transport of drugs loaded in the magnetogels into Small Unilamellar Vesicles (SUVs), used as membrane models, was assessed. For that purpose, FRET assays were employed, the SUVs being labelled with the lipid probe Nile Red (acceptor), and the drugs acting as energy donors.

The magnetogel possessing the RGD sequence, while being suitable for target therapy, was the most promising for delivery of the thienopyridine derivative, while all the three magnetogels were suitable for the delivery of curcumin.

Considering the antitumor capabilities of both drugs and the biocompatibility of the gels, the proof-of-concept presented in this work indicates that these systems are promising for future developments of dual oncological therapy, by combining magnetic hyperthermia and drug delivery.

\section{Conflicts of interest}

There are no conflicts of interest to declare.

\section{Acknowledgements}

This work was supported by the Portuguese Foundation for Science and Technology (FCT) in the framework of the Strategic Funding of CF-UM-UP (UID/FIS/04650/2013) and CQUM (UID/QUI/00686/ /2016). FCT, FEDER, PORTUGAL2020 and COMPETE2020 are also acknowledged for funding under research projects PTDC/QUIQFI/28020/2017 (POCI-01-0145-FEDER-028020) and PTDC/QUIQOR/29015/2017 (POCI-01-0145-FEDER-029015). S.R.S.V. and A.R.O.R. acknowledge FCT for research grants under UID/FIS/04650/2013 funding.

Authors gratefully acknowledge $\mathrm{Dr}$ L. M. Vieira for carrying out the FTIR measurements at the Infrared Spectroscopy Lab. of the Centre of Physics, Univ. Minho.

\section{Notes and references}

1 T. K. Indira and P. K. Lakshmi, Magnetic Nanoparticles - A Review, Int. J. Pharm. Sci. Nanotech., 2010, 3, 1035-1042.
2 A. R. O. Rodrigues, J. M. F. Ramos, I. T. Gomes, B. G. Almeida, J. P. Araújo, M.-J. R. P. Queiroz, P. J. G. Coutinho and E. M. S. Castanheira, Magnetoliposomes based on manganese ferrite nanoparticles as nanocarriers for antitumor drugs, RSC Advances, 2016, 6, 17302-17313.

3 B. Issa, I. M. Obaidat, B. A. Albiss and Y. Haik, Magnetic Nanoparticles: Surface Effects and Properties Related to Biomedicine Applications, Int. J. Mol. Sci., 2013, 14, 2126621305.

4 N. R. Datta, S. Krishnan, D. E. Speiser, E. Neufeld, N. Kuster, S. Bodis and H. Hofmann, Magnetic nanoparticle-induced hyperthermia with appropriate payloads: Paul Ehrlich's "magic (nano)bullet" for cancer theranostics?, Cancer Treat. Rev., 2016, 50, 217-227.

5 C. Pereira, A. M. Pereira, C. Fernandes, M. Rocha, R. Mendes, M. P. F.-Garcia, A. Guedes, P. B. Tavares, J.-M. Grenèche, J. P. Araújo and C. Freire, Superparamagnetic $\mathrm{MFe}_{2} \mathrm{O}_{4}(\mathrm{M}=\mathrm{Fe}$, Co, $\mathrm{Mn}$ ) Nanoparticles: Tuning the Particle Size and Magnetic Properties through a Novel One-Step Coprecipitation Route, Chem. Mater., 2012, 24, 1496-1504.

6 D. Carta, M. F. Casula, P. Floris, A. Falqui, G. Mountjoy, A. Boni, C. Sangregorio and A. Corrias, Synthesis and microstructure of manganese ferrite colloidal nanocrystals, Phys. Chem. Chem. Phys. 2010, 12, 5074-5083.

7 L. I. Cabrera, A. Somoza, J. F. Marco, C. J. Serna and M. P. Morales, Synthesis and surface modification of uniform $\mathrm{MFe}_{2} \mathrm{O}_{4}$ ( $\mathrm{M}=\mathrm{Fe}, \mathrm{Mn}$, and $\mathrm{Co}$ ) nanoparticles with tunable sizes and functionalities, J. Nanoparticle Res., 2012, 14, 1-14.

8 M. Colombo, S. Carregal-Romero, M. F. Casula, L. Gutiérrez, M. P. Morales, I. B. Böhm, J. T. Heverhagen, D. Prosperi and W. J. Parak, Biological applications of magnetic nanoparticles, Chem. Soc. Rev., 2012, 41, 4306-4334.

9 L. Kafrouni and O. Savadogo, Recent progress on magnetic nanoparticles for magnetic hyperthermia, Prog. Biomater., 2016, 5, 147-160.

10 A. R. O. Rodrigues, B. G. Almeida, J. M. Rodrigues, M.-J. R. P. Queiroz, R. C. Calhelha, I. C. F. R. Ferreira, A. Pires, A. M. Pereira, J. P. Araújo, P. J. G. Coutinho and E. M. S. Castanheira, Magnetoliposomes as carriers for promising antitumor thieno[3,2-b]pyridin-7-arylamines: photophysical and biological studies, RSC Advances, 2017, 7, 15352-15361.

11 Z. K. Zhang and S. Q. Song, Thermosensitive/superparamagnetic iron oxide nanoparticle-loaded nanocapsule hydrogels for multiple cancer hyperthermia, Biomaterials, 2016, 106, 13-23.

12 X. Li, J. Wei, K. E. Aifantis, Y. Fan, Q. Feng, F. Z. Cui and F. Watari, Current investigations into magnetic nanoparticles for biomedical applications, J. Biomed. Mater. Res. A, 2016, 104, 1285-1296.

13 T. R. Hoare and D. Kohane, Hydrogels in drug delivery: Progress and challenges, Polymer, 2008, 49, 1993-2007.

14 M. Ma, Y. Kuang, Y. Gao, Y. Zhang, P. Gao and B. Xu, Aromaticaromatic interactions induce the self-assembly of pentapeptidic derivatives in water to form nanofibers and supramolecular hydrogels, J. Am. Chem. Soc., 2010, 132, 2719-2728.

15 L. Chen, S. Revel, K. Morris, L. C. Serpell and D. J. Adams, Effect of Molecular Structure on the Properties of NaphthaleneDipeptide Hydrogelators, Langmuir, 2010, 26, 13466-13471.

16 S. Sutton, N. L. Campbell, A. I. Cooper, M. Kirkland, W. J. Frith and D. J. Adams, Controlled release from modified amino acid 
hydrogels governed by molecular size or network dynamics, Langmuir, 2009, 25, 10285-10291.

17 M. McKenzie, D. Betts, A. Suh, K. Bui, L. D. Kim and H. Cho, Hydrogel-Based Drug Delivery Systems for Poorly WaterSoluble Drugs, Molecules, 2015, 20, 20397-20408.

18 E. Caló and V. V. Khutoryanskiy, Biomedical applications of hydrogels: A review of patents and commercial products, Eur. Polym. J., 2015, 65, 252-267.

19 H. Vilaça, A. C. L. Hortelão, E. M. S. Castanheira, M.-J. R. P. Queiroz, L. Hilliou, I. W. Hamley, J. A. Martins and P. M. T. Ferreira, Dehydrodipeptide hydrogelators containing naproxen $\mathrm{N}$-capped Tryptophan: self-assembly, hydrogel characterization and evaluation as potential drug nanocarriers, Biomacromolecules, 2015, 16, 3562-3573.

20 A. Z. Cardoso, A. E. A. Alvarez, B. N. Cattoz, P. C. Griffiths, S. M. King, W. J. Frith and D. J. Adams, The influence of the kinetics of self-assembly on the properties of dipeptide hydrogels, Faraday Disc., 2013, 166, 101-116.

21 G. Fichman and E. Gazit, Self-assembly of short peptides to form hydrogels: design of building blocks, physical properties and technological applications, Acta Biomaterialia, 2014, 10, 16711682.

22 J. Li, Y. Kuang, Y. Gao, X. Du, J. Shi and B. Xu, D-amino acids boost the selectivity and confer supramolecular hydrogels of a nonsteroidal anti-inflammatory drug (NSAID), J. Am. Chem. Soc., 2013, 135, 542-545.

23 H. Vilaça, G. Pereira, T. G. Castro, B. F. Hermenegildo, J. Shi, T. Q. Faria, N. Micaêlo, R. M. M. Brito, B. Xu, E. M. S. Castanheira, J. A. Martins and P. M. T. Ferreira, New self-assembled supramolecular hydrogels based on dehydropeptides, J. Mater. Chem. B, 2015, 3, 6355-6367.

24 H. Vilaça, T. Castro, F. M. G. Costa, M. Melle-Franco, L. Hilliou, I. W. Hamley, E. M. S. Castanheira, J. A. Martins and P. M. T. Ferreira, Self-assembled dehydropeptide RGD hydrogels for drug delivery applications, J. Mater. Chem. B, 2017, 5, 86078617.

25 E. Ruoslahti, RGD and other recognition sequences for integrins, Ann. Rev. Cell Develop. Biol., 1996, 12, 697-715.

26 A. Akbarzadeh, M. Samiei and S. Davaran, Magnetic nanoparticles: preparation, physical properties, and applications in biomedicine, Nanoscale Res. Lett., 2012, 7, 144-157.

27 A. Hervault and N. T. K. Thanh, Magnetic nanoparticle-based therapeutic agents for thermo-chemotherapy treatment of cancer, Nanoscale, 2014, 6, 11553-11573.

28 M.-J. R. P. Queiroz, R. C. Calhelha, L. Vale-Silva, E. Pinto and M. S.-J. Nascimento, Novel [6-(hetero)arylamino]thieno [3,2-b]pyridines: synthesis and antitumoral activities, Eur. J. Med. Chem., 2010, 45, 5732-5738.

29 C. N. C. Costa, A. C. L. Hortelão, J. M. F. Ramos, A. D. S. Oliveira, R. C. Calhelha, M.-J. R. P. Queiroz, P. J. G. Coutinho and E. M. S. Castanheira, A new antitumoral heteroarylaminothieno[3,2-b]pyridine derivative: its incorporation into liposomes and interaction with proteins monitored by fluorescence, Photochem. Photobiol. Sci., 2014, 13, 17301740.

30 B. B. Aggarwal and K. B. Harikumar, Potential therapeutic effects of curcumin, the anti-inflammatory agent, against neurodegenerative, cardiovascular, pulmonary, metabolic, autoimmune and neoplastic diseases, Int. J. Biochem. Cell Biol., 2009, 41, 40-59.

31 B. B. Aggarwal and B. Sung, Pharmacological basis for the role of curcumin in chronic diseases: an age-old spice with modern targets, Trends Pharmacol. Sci., 2009, 30, 85-94.

32 A. Goel, A. B. Kunnumakkara and B. B. Aggarwal, Curcumin as 'Curecumin': from kitchen to clinic, Biochem. Pharmacol., 2008, 75, 787-809.

33 P. Anand, A. B. Kunnumakkara, R. A. Newman and B. B. Aggarwal, Bioavailability of curcumin: Problems and promises, Mol. Pharm., 2007, 4, 807-818.

34 S. Bisht, G. Feldmann, S. Soni, R. Ravi, C. Karikar, A. Maitra and A. Maitra, Polymeric nanoparticle-encapsulated curcumin ('nanocurcumin'): a novel strategy for human cancer therapy, J. Nanobiotech., 2007, 5, article 3.

35 D. Perrin and B. Dempsey, Buffers for $\mathrm{pH}$ and Metal Ion Control, London, England, Chapman and Hall, 1974.

36 J. M. H. Kremer, M. W. J. v. d. Esker, C. Pathmamanoharan and P. H. Wiersema, Vesicles of variable diameter prepared by a modified injection method, Biochemistry, 1977, 16, 3932-3935.

37 P. Greenspan and S. Fowler, Spectrofluorometric studies of the lipid probe Nile Red, J. Lipid Res., 1985, 26, 781-789.

38 I. Krishnamoorthy and G. Krishnamoorthy, Probing the link between proton transport and water content in lipid membranes, J. Phys. Chem. B, 2001, 105, 1484-1488.

39 P. J. G. Coutinho, E. M. S. Castanheira, M. C. Rei and M. E. C. D. R. Oliveira, Nile Red and DCM fluorescence anisotropy studies in $\mathrm{C}_{12} \mathrm{E}_{7}$ /DPPC Mixed Systems, J. Phys. Chem. B, 2002, 106, 1284112846.

40 G. A. Crosby and J. N. Demas, The measurement of photoluminescence quantum yields. - Review, J. Phys. Chem., 1971, 75, 991-1024.

$41 \mathrm{~S}$. Fery-Forgues and D. Lavabre, Are fluorescence quantum yields so tricky to measure? A demonstration using familiar stationery products, J. Chem. Educ., 1999, 76, 1260-1264.

42 E. P. Kirby and R. F. Steiner, Influence of solvent and temperature upon the fluorescence of indole derivatives, J. Phys. Chem., 1970, 74, 4480-4490.

43 B. Valeur, Molecular fluorescence - Principles and Applications, Wiley-VCH, Weinheim, 2001.

44 Gaussian 09, Revision A.02, M. J. Frisch, G. W. Trucks, H. B. Schlegel, G. E. Scuseria, M. A. Robb, J. R. Cheeseman, G. Scalmani, V. Barone, B. Mennucci, G. A. Petersson, H. Nakatsuji, M. Caricato, X. Li, H. P. Hratchian, A. F. Izmaylov, J. Bloino, G. Zheng, J. L. Sonnenberg, M. Hada, M. Ehara, K. Toyota, R. Fukuda, J. Hasegawa, M. Ishida, T. Nakajima, Y. Honda, O. Kitao, H. Nakai, T. Vreven, J. A. Montgomery Jr., J. E. Peralta, F. Ogliaro, M. Bearpark, J. J. Heyd, E. Brothers, K. N. Kudin, V. N. Staroverov, R. Kobayashi, J. Normand, K. Raghavachari, A. Rendell, J. C. Burant, S. S. Iyengar, J. Tomasi, M. Cossi, N. Rega, J. M. Millam, M. Klene, J. E. Knox, J. B. Cross, V. Bakken, C. Adamo, J. Jaramillo, R. Gomperts, R. E. Stratmann, O. Yazyev, A. J. Austin, R. Cammi, C. Pomelli, J. W. Ochterski, R. L. Martin, K. Morokuma, V. G. Zakrzewski, G. A. Voth, P. Salvador, J.J. Dannenberg, S. Dapprich, A. D. Daniels, 
Ö. Farkas, J. B. Foresman, J. V. Ortiz, J. Cioslowski, D. J. Fox, Gaussian, Inc., Wallingford CT, 2009.

45 H. Azizian, Z. Mousavi, H. Faraji, M. Tajik, K. Bagherzadeh, P. Bayat, A. Shafiee and A. Almasirad, Arylhydrazone derivatives of naproxen as new analgesic and antiinflammatory agents: Design, synthesis and molecular docking studies, J. Mol. Graph. Model.., 2016, 67, 127-136.

46 L. Benco, D. Tunega, J. Hafner and H. Lischka, Upper limit of the $\mathrm{O}-\mathrm{H} \cdots \mathrm{O}$ hydrogen bond. Ab initio study of the kaolinite structure, J. Phys. Chem. B, 2001, 105, 10812-10817.

47 M. Y. Rafique, P. Li-Qing, Q. Javed, M. Z. Iqbal, Q. Hong-Mei, M. H. Farooq, G. Zhen-Gang and M. Tanveer, Growth of monodisperse nanospheres of $\mathrm{MnFe}_{2} \mathrm{O}_{4}$ with enhanced magnetic and optical properties, Chinese Phys. B, 2013, 22, article 107101.

48 L. Liu and H. Gao, Quantum chemistry study of molecular structure and vibrational spectrum of naproxen, Spectrochim. Acta A, 2012, 86, 131-138.

$49 \mathrm{M}$. Wolpert and P. Hellwig, Infrared spectra and molar absorption coefficients of the 20 alpha amino acids in aqueous solutions in the spectral range from 1800 to 500 $\mathrm{cm}^{-1}$, Spectrochim. Acta A, 2006, 64, 987-1001.

$50 \mathrm{~S}$. Y. Lin and H. L. Chu, Fourier transform infrared spectroscopy used to evidence the prevention of $\beta$-sheet formation of amyloid $\beta(1-40)$ peptide by a short amyloid fragment, Int. J. Biol. Macromol., 2003, 32, 173-177.

51 H. Fabian, W. Mäntele and J. M. Chalmers, Infrared Spectroscopy of Proteins, in Handbook of Vibrational Spectroscopy, 2006, J. M. Chalmers and P. R. Griffiths Eds., Wiley-Interscience, pp. 3999-3454.

52 H. Fabian and C. P. Schultz, Fourier Transform Infrared Spectroscopy in Peptide and Protein Analysis, in
Encyclopedia of Analytical Chemistry, 2006, R. A. Meyers and C. Schöneich Eds., John Wiley \& Sons, pp. 1-25.

53 B. H. Stuart, Infrared spectroscopy of biological applications: An overview, in Encyclopedia of Analytical Chemistry, 2012, R. A. Meyers Ed., John Wiley \& Sons, pp. 1-17.

54 A. Barik, B. Mishra, A. Kunwar and K. I. Priyadarsini, Interaction of curcumin with human serum albumin: Thermodynamic properties, fluorescence energy transfer and denaturation effects, Chem. Phys. Lett., 2007, 436, 239243.

55 A. S. Abreu, E. M. S. Castanheira, M.-J. R. P. Queiroz, P. M. T. Ferreira, L. A. Vale-Silva and E. Pinto, Nanoliposomes for encapsulation and delivery of the potential antitumoral methyl 6-methoxy-3(4-methoxyphenyl)-1H-indole-2-carboxylate, Nanoscale Res. Lett., 2011, 6, article 482.

56 C. Toniolo, M. Crisma, F. Formaggio, C. Peggion, V. Monaco, C. Goulard, S. Rebuffat and B. Bodo, Effect of $\mathrm{N}^{\alpha}$-Acyl chain length on the membrane-modifying properties of synthetic analogs of the lipopeptaibol Trichogin GA IV, J. Am. Chem. Soc., 1996, 118, 4952-4958.

57 M. Lucaciu, S. Rebuffat, C. Goulard, H. Duclohier, G. Molle and B. Bodo, Interaction of the 14-residue peptaibols, harzianins $\mathrm{HC}$, with lipid bilayers: permeability modifications and conductance properties, Biochim. Biophys. Acta, 1997, 1323, 85-96.

58 S. R. Meech and D. Phillips, Photophysics of some common fluorescence standards, J. Photochem., 1983, 23, 193-217.

59 D. Magde, R. Wong and P. G. Seybold, Fluorescence quantum yields and their relation to lifetimes of rhodamine $6 \mathrm{G}$ and fluorescein in nine solvents: improved absolute standards for quantum yields, Photochem. Photobiol., 2002, 75, 327-334.

60 H. Curtis and N. S. Barnes, Biology, 5th ed., Worth Publishers, New York, USA, 1989, part 1. 